\title{
Microneedle Characterization Using a Double-Layer Skin Simulant
}

\author{
W. W. Koelmans ${ }^{1,4}$, G. Krishnamoorthy ${ }^{1}$, A. Heskamp 2 , J. Wissink ${ }^{2}$, S. Misra ${ }^{3} \&$ N. Tas ${ }^{1}$ \\ ${ }^{1} \mathrm{MESA}^{+}$Institute for Nanotechnology, University of Twente, Enschede, The Netherlands \\ ${ }^{2}$ U-Needle B.V., Enschede, The Netherlands \\ ${ }^{3}$ MIRA Institute for Biomedical Technology and Technical Medicine, University of Twente, Enschede, The Nether- \\ lands \\ ${ }^{4}$ Currently at IBM Research-Zürich, Säumerstrasse 4, 8803 Rüschlikon, Switzerland \\ Correspondence: Wabe W. Koelmans, IBM Research-Zürich, Säumerstrasse 4, 8803 Rüschlikon, Switzerland. \\ E-mail: w.w.koelmans@alumnus.utwente.nl
}

\author{
Received: May 9, 2013 Accepted: June 10, 2013 Online Published: July 17, 2013 \\ doi:10.5539/mer.v3n2p51 URL: http://dx.doi.org/10.5539/mer.v3n2p51
}

The research is supported by the Dutch Ministry of Economic Affairs, region Overijssel, region Gelderland, under the programme Nano4 Vitaliy

\begin{abstract}
Microneedles are important injecting devices for the delivery of pharmaceutical drugs in precise amounts at specific locations. To optimize the design of microneedles, a thorough understanding of the needle skin interaction has to be obtained. We present methods to characterize the mechanical interaction between microneedles and a double-layer skin simulant. The methods are applied to silicon microneedles designed (fabricated in-house) and to commercially available hypodermic needles (as a reference). The skin simulant consists of an agarose gel and a layer of commercially available needle-testing foil to mimic the dermis and the stratum corneum, respectively. A systematic analysis of the behaviour of a silicon microneedle inside the skin simulant is presented and compared with that of current hypodermic needles. The microneedle geometry is related to the force curves measured, with the help of (environmental) scanning electron and confocal microscope images that visualize the microscale physical phenomena that occur inside the skin simulant. We measure a $12 \%$ and $14 \%$ increase in insertion force when increasing the insertion speed from 100 to $500 \mathrm{\mu ms}^{-1}$ for hypodermic needles and microneedles, respectively. The characterization methods are shown to be complementary to in vivo experiments and enable advanced imaging techniques and can significantly help the design of microneedles.
\end{abstract}

Keywords: microneedles, insertion force, skin simulant, hypodermic needle, vaccination, confocal microscopy, force curves, characterization method

\section{Introduction}

Microneedles are becoming increasingly important in the delivery of drugs since their introduction in the early 1990s (Campbell, 1991). Microneedles offer two distinct advantages over conventional hypodermic needles. First, microneedles are less invasive when used for the injection of drugs or removal of bodily fluids, leading to reduced patient trauma (Doraiswamy, 2010). The limited length of the microneedle is known to significantly reduce pain (Gill, 2008). Second, microneedles enable accurate site-specific drug-delivery, e.g., for injection of drugs into the eyes (Jiang, 2007).

Microneedles are developed for a variety of drug-delivery applications (Donnelly, 2010; Park, 2005; Stoeber, 2005). These can be divided into two categories: hollow and solid microneedles. Hollow microneedles feature microchannels through which drugs are delivered (Roxhed, 2007; Zhang, 2009a). The drug injection can be initiated by means of a syringe (Wang, 2006). Alternatively a (implantable) system containing a reservoir and a (manual) pump (Hilt, 2005) can be used. The delivery mechanism is generally based on forced convection.

Solid microneedles are micro-structured projections that either serve to increase skin permeability by the formation 
of conduits in the stratum corneum (Henry, 1998; McAllister, 2003) or to directly inject drugs by coating the projection prior to insertion (Gill, 2007). The tip is inserted into the skin to provide intra-dermal delivery of active agents, which otherwise would not cross the stratum corneum. The delivery mechanism is based on diffusion. The drug molecules attached get released within the epidermis, where they can readily reach their site of action. An alternative method exists in which the drug is encapsulated in a biodegradable polymer. The polymer encapsulating the drug is inserted into the skin, and, depending on the polymer dissolving time, the drug is released (Park, 2006; Lee, 2008).

To increase the diffusion and convection rates for both hollow and solid microneedles, the needles can be manufactured in arrays (Gill, 2007). Arrays combined with transdermal patches dramatically increase the types of drugs that can be delivered using patches (McAllister, 2003). An array of 400 microneedles was demonstrated to pierce human skin while delivering drug molecules (Hilt, 2005). Arrays are also used for the removal of fluid from the body for diagnostics, such as blood glucose measurements (Wang, 2005).

The microneedle designs include conical and beveled tips, and have, in the case of hollow microneedles, an opening at the top, side or a mix of top and side opening (Roxhed, 2007).

Microneedles have been fabricated out of a variety of materials, such as metal, silicon, silicon dioxide, polymers, and glass (McAllister, 2003; Kim, 2006e). Silicon is an excellent material for needle fabrication, because it is strong, wear resistant, and not susceptible to fatigue. Micromachining the needles using cleanroom massfabrication techniques enables more accurate control over the needle dimensions than conventional types of machining, such as grinding and laser cutting do (Gill, 2007).

Despite the potential that microneedles hold, detailed experimental studies of the microneedle's interaction with skin tissue are scarce. In particular, the forces required to insert microneedles into the skin have to be quantified and related to the microneedle design. Understanding the origin of insertion forces helps optimize microneedle design. A high insertion force can lead to breaking of individual needles, limiting the number of microneedles in an array (Davis, 2004). Also, the insertion force directly relates to pain during the insertion process (Egekvist, 1999). A measurement of the insertion force versus the displacement provides valuable information about the mechanical behaviour of a needle.

In this paper, we introduce methods to characterize the interaction between microneedles and soft tissue. Using these methods we perform a careful and systematic experimental analysis of the needle-tissue interaction force curve. Next, we relate the force characteristics to observations from several imaging techniques, such as environmental SEM and confocal microscopy. The experimental studies are performed with different needle designs and under different insertion conditions, demonstrating the versatility of the approach. All experiments are performed on a double-layer skin simulant that consists of an agarose gel and a polyurethane foil. The gel and foil act as dermis and stratum corneum, respectively. The use of a skin simulant holds several advantages. The repeatability of experiments is improved because of the high degree of control in skin-simulant preparation. The experiments can be conducted in a controlled manner and are more readily observable by microscopy than in vivo experiments. Furthermore, skin simulants offer a significant cost advantage over experiments on live humans or on prepared skin samples from either human or animal cadavers.

In this study, we have used solid silicon microneedles and commercially available hypodermic needles (the latter as a reference). From here on the term microneedle refers to a solid silicon microneedle unless specifically mentioned otherwise. First, various concentrations of agarose gels (1\%-4\%) are tested to match the properties of the dermis. Second, insertion force experiments are performed with both hypodermic needles and microneedles into the agarose gel, and third, into the double-layer skin simulant consisting of a polyurethane foil on 3\% agarose gel. We identify the different phases of the insertion force curve that that stem from the specific needle shape and geometry, present a range of images of the needles interacting with the soft-tissue simulants, and finally draw conclusions.

\section{Experimental Details}

\subsection{Microneedle Fabrication}

The silicon microneedle design used in this study is shown in Figure 1(a). The microneedle is etched out of a silicon $\langle 110\rangle$ wafer in such a way that the $z$-direction (see Figure 1 (b) for the definition of the $z$-direction) is oriented parallel to the wafer surface (in-plane fabrication). Given this orientation, the microneedle length can be freely chosen. Lengths of upto $10 \mathrm{~mm}$ have been fabricated and successfully tested. 
The microneedle is fabricated using wet anistropic etching on either side of the wafer. First, on both the front and the back side of the wafer, a hard etch mask is created by photolithography. While the top side is being anisotropically etched, the bottom side of the wafer is passivated. After the first etching step (through the wafer), the exposed $\langle 111\rangle$ planes are passivated to keep them from etching any further. Next, the bottom side of the wafer is anistropically etched, after removing the passivation layer on that side by plasma reactive ion etching. After these etching steps, the masking layers are removed, the microneedles are released from the wafer and assembled in standard needle hubs (see the right inset in Figure ).

In Figure 1(b) a schematic drawing of the needle is shown. The dimensions in Figure 1(b) are the mean values of optical microscopy measurements of seven needles (relative standard deviation below 1\%). The bevel plane, which is marked by the points $Z_{0}, Z_{1}, Z_{2}$ and $Z_{3}$, is a slow-etching $\langle 111\rangle$ crystal plane. The sides of the shaft are two $\langle 111\rangle$ planes and two $\langle 110\rangle$ planes. The cross section of the shaft has a rectangular shape with a dimension of approximately $300 \mu \mathrm{m} \times 400 \mu \mathrm{m}$. For comparison, a photograph and a SEM image of a hypodermic needle are shown in Figures 1(c) and 1(d), respectively. The hypodermic needle is a 30G needle, type BD Microlance 3 (Becton, Dickinson and Company, Franklin Lakes, USA) with a length of $13 \mathrm{~mm}$ and a diameter of $0.3 \mathrm{~mm}$. The tip of the needle is marked by $Z_{0}^{\prime}$, the transition from the upper to the lower bevel plane by $Z_{1}^{\prime}$, and the transition of the lower bevel plane to the shaft $Z_{2}^{\prime}$.

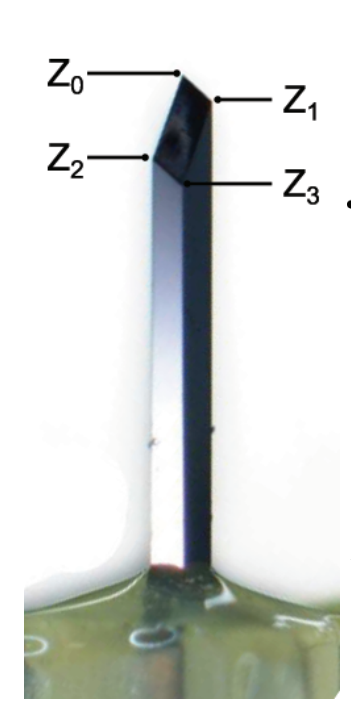

(a)

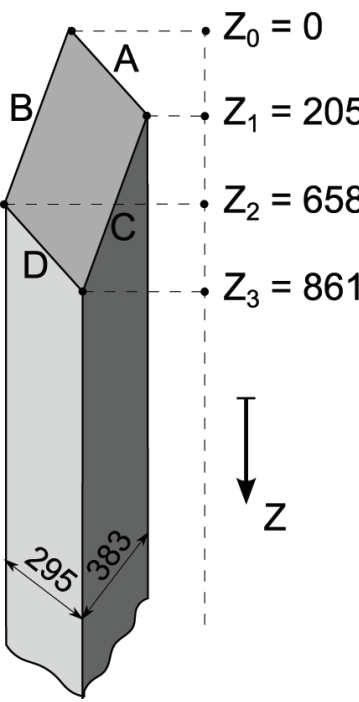

(b)

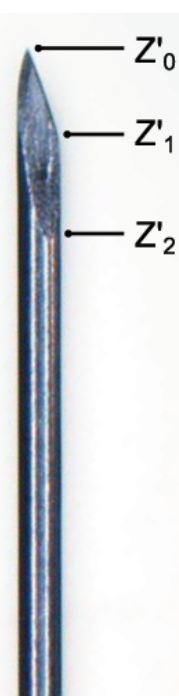

(c)

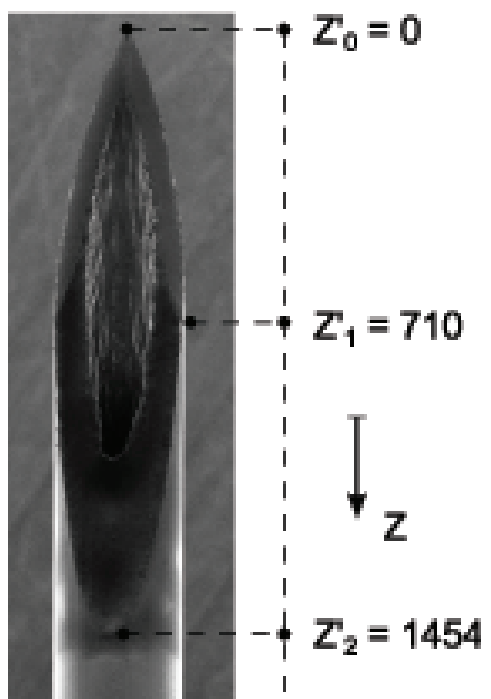

(d)

Figure 1. (a) Microscope image of the microneedle. The corners of the bevel plane are marked $Z_{0}$ (tip), $Z_{1}, Z_{2}$ and $Z_{3}$. (b) Microneedle geometry with dimensions in micrometers. The sides of the bevel plane are marked A, B, C and D. (c) Microscope image of a 30G hypodermic needle. (d) SEM image of the tip of a 30G needle with dimensions in micrometers

\subsection{Needle-Insertion Setup}

Figure 2 shows the setup used in this study. In this setup, the needle position does not change to facilitate imaging with a confocal microscope. The experiment is performed by translating the skin simulant towards the needle while recording the force in the $z$-direction with the force sensor at the base of the needle. The force sensor is a six-axis Nano17 force-torque sensor (ATI Industrial Automation, Apex, USA) with a force and torque resolution of $3.1 \mathrm{mN}$ and $15.6 \mathrm{mN} \mathrm{mm}$, respectively. To increase the resolution, each data point is an average of 4000 force readings. Unless stated otherwise, each insertion experiment is repeated three times with three different needles adding upto a total of nine experiments per data point. We translated the skin simulant in $y$-direction in between each insertion experiment to obtain a new insertion site. In all experiments reported we show the absolute force in $z$-direction. The forces in the $x$ - and $y$-directions and the torques are minimized by careful alignment of the skin simulant with respect to the needle. Note that the insertion depths used in the experiments are larger than in many microneedle applications (e.g., vaccination). A large insertion depth was used for the sake of completeness of the study. 


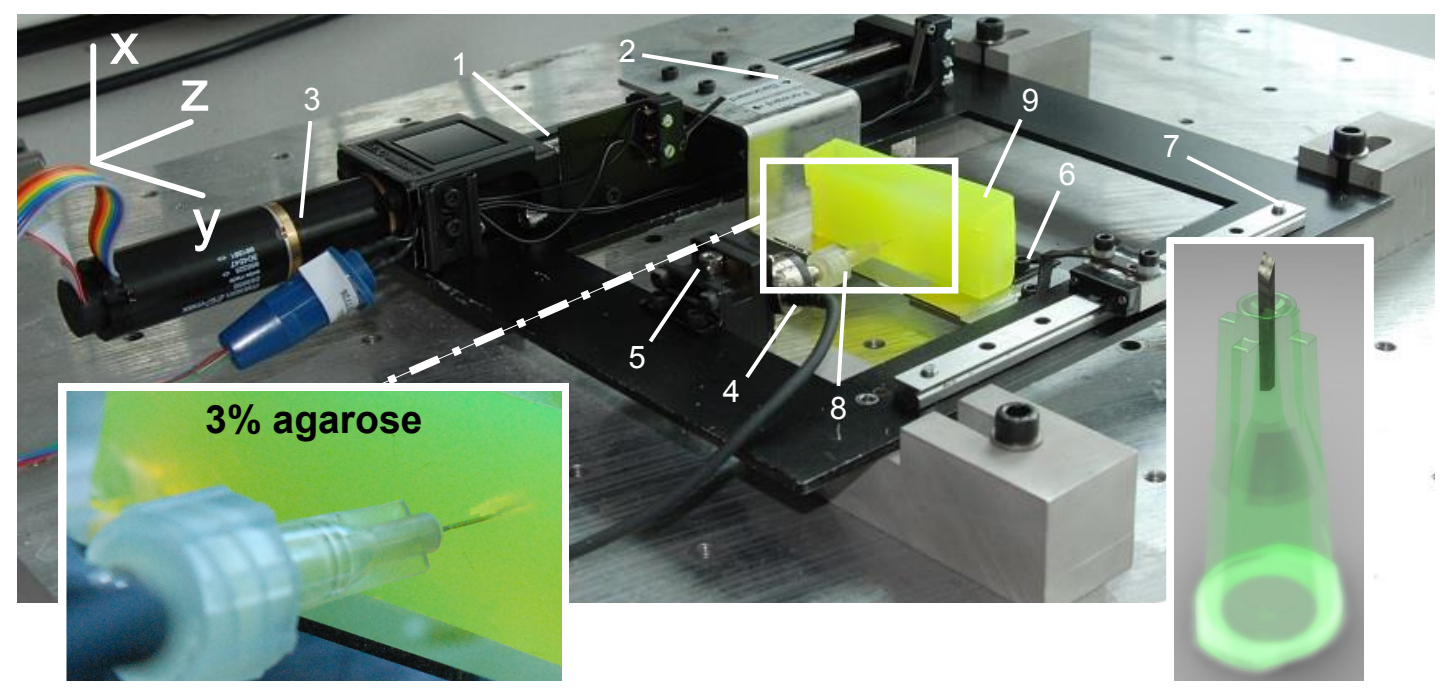

Figure 2. Photograph of the insertion force-displacement measurement setup. The important parts are marked: 1) the linear actuator, 2) carriage for holding samples, 3) EC-motor driving the linear actuator, 4) force sensor, 5) adjustable stage to alter the position of the needle in the $x$-direction, 6) braces to hold sample in place, 7) linear guide to prevent bending and motion of the carriage in $x$-direction, 8) needle, and 9) sample. The left inset shows a $30 \mathrm{G}$ needle inside the $3 \%$ agarose gel. The right inset shows drawing of the assembly of the microneedle in a standard needle hub

\subsection{Microscopy}

We used of two kinds of microscopic imaging modalities. The first is a confocal microscope, a Laser Scanning Microscope LSM 510 (Carl-Zeiss, Sliedrecht, the Netherlands). The imaging plane is $y z$; needle insertion is along the $z$-axis. The needle insertion was visualized with differential interference contrast (DIC) and epifluorescence using a 488-nm argon laser and a low-pass filter with cut-off at $505 \mathrm{~nm}$.

The second microscope is a Scanning Electron Microscope Quanta 450 (FEI Company, Eindhoven, the Netherlands). This SEM has an environmental option (E-SEM) that allows wet and/or contaminated samples to be imaged. In this mode, a water vapor is led into the chamber at a pressure of around $1000 \mathrm{~Pa}$. We used this mode to image the wet agarose gel.

\subsection{Agarose Preparation}

We prepared agarose gel, which can be fine-tuned to resemble part of the stress-strain relation of skin by varying the agarose concentration. Agarose powder (Sigma Aldrich, Zwijndrecht, Netherlands) was dissolved in $10 \times$ tris acetate EDTA (TAE) buffer. The mixture was heated until the agarose powder was completely dissolved. We added $100 \mu 1$ of $1 \mathrm{mM}$ sodium fluorescein solution to $100 \mathrm{ml}$ of the mixture for epifluorescent imaging. After cooling the mixture under running tap water, it was poured into a mould and left at room temperature for $10 \mathrm{~min}$ for the gel to solidify. After solidification, the gel is ready for use. The left inset of Figure 2 shows the 3\% (mass) agarose gel prepared. In the case of the double-layer system, the polyurethane foil was placed on the mould prior to pouring the gel. The polyurethane foil is a needle-testing foil called Dekatest (Melab, Leonberg, Germany) and has a thickness of $80 \mu \mathrm{m}$.

\subsection{Agarose Characterization}

To choose the right agarose concentration to model the human skin, the stress-strain relation was determined from a compression test using a rheometer (Physica MCR501, Anton Paar, Ashland, USA). A circularly shaped sample of the agarose gel, with a diameter of $25 \mathrm{~mm}$ and a thickness of $6 \mathrm{~mm}$ was prepared. We extracted the Young's modulus from the linear part of the stress-strain curve. See the inset of Figure 3 for an example of a stress-strain curve obtained on $3 \%$ agarose gel.

\section{Results and Discussion}

Two different skin simulants have been tested, namely, plain agarose gel and, polyurethane foil supported by $3 \%$ agarose gel. 


\subsection{Single-Layer System-Agarose Gel}

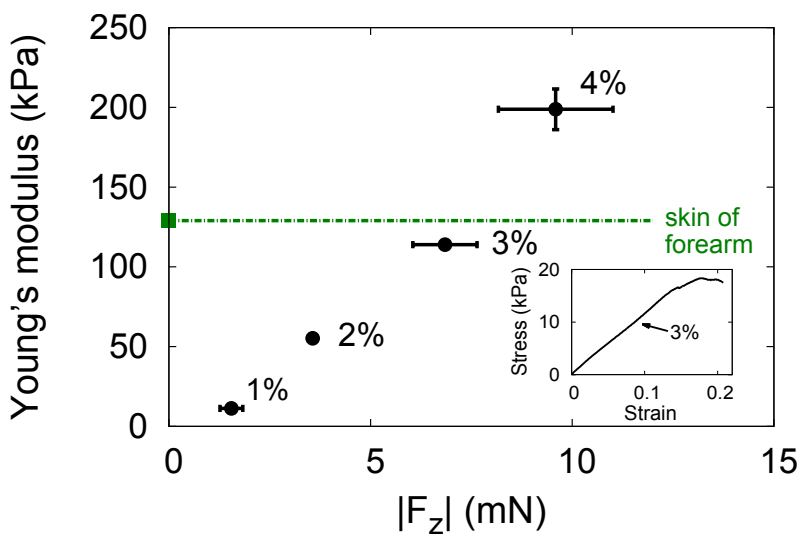

Figure 3. Agarose gel characterization using $30 \mathrm{G}$ needles. Young's modulus is plotted versus the insertion force at $3 \mathrm{~mm}$ needle displacement for different agarose concentrations. The data points represent the mean value of the

experiment repeated nine times, with the error bars being standard deviation. The dashed line indicates the

Young's modulus values of skin of the forearm. The inset shows an example of a stress-strain curve measured on the $3 \%$ agarose gel
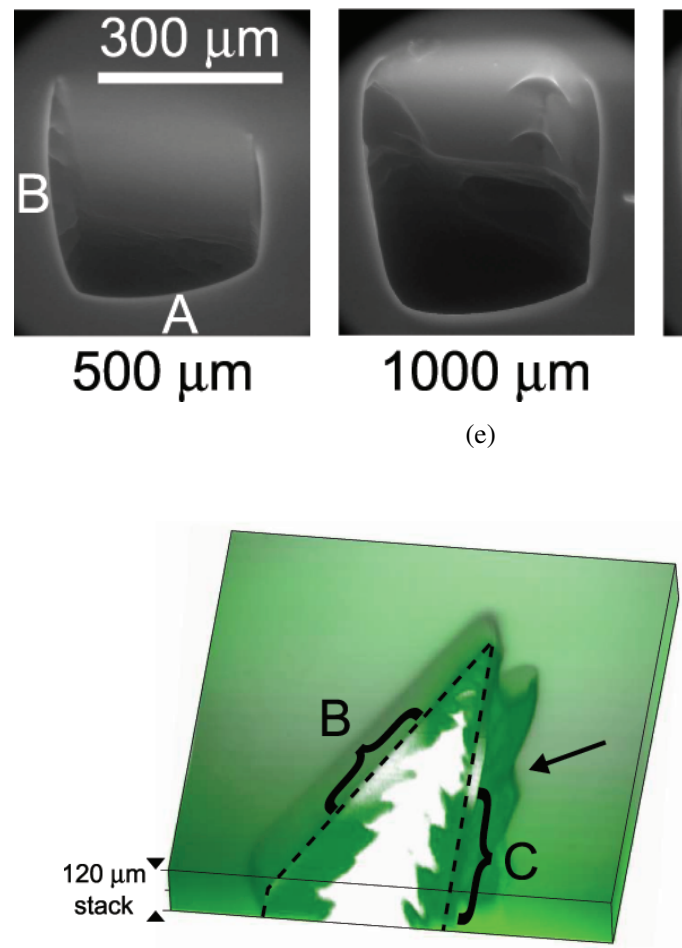

(f)

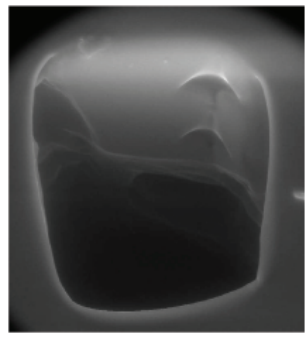

$1000 \mu \mathrm{m}$

(e)

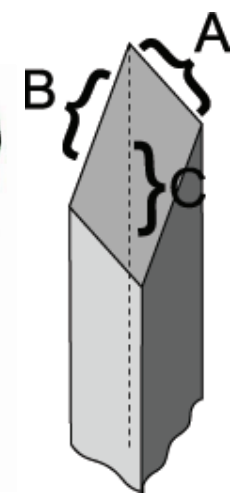

(g)

Figure 4. (a) Environmental SEM images of microneedle insertion holes in agarose gel at identical scale and orientation. The images shown are top view of the gel with the insertion direction into the page. The distance under each image corresponds to the displacement of the needle into the agarose gel. The white arrow indicates a mode-I crack (opening). (b) A 3D confocal microscopy image of the agarose gel surrounding the microneedle.

The stack contains 20 images and is $120 \mu \mathrm{m}$ high. The black arrow indicates a mode-II crack (rupture). The dotted line indicates the contour of the needle. (c) A schematic representation of the microneedle defining sides $\mathrm{A}, \mathrm{B}$ and $\mathrm{C}$ 
The Young's moduli of four samples of agarose gel with concentrations of $1 \%, 2 \%, 3 \%$ and $4 \%$ (mass) have been measured using the rheometer. Also, needle insertion experiments have been performed on each sample using the $30 \mathrm{G}$ needle. In Figure 3, the insertion force at 3-mm needle displacement is plotted versus Young's modulus for the various agarose concentrations. The insertion speed was set to $250 \mu \mathrm{m} \mathrm{s}^{-1}$. We selected the $3 \%$ agarose gel because its Young's modulus (114 kPa) is similar to that of the human forearm (129 kPa) (Diridollou, 2000).

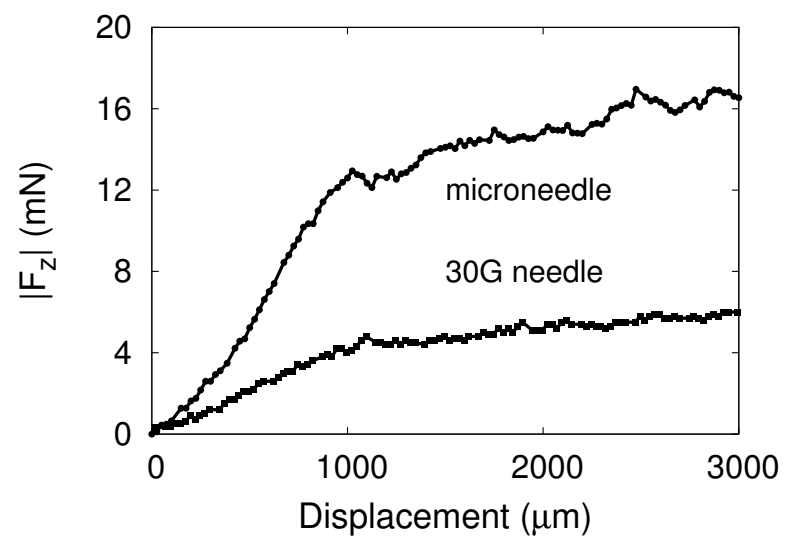

Figure 5. Insertion-force curves of a microneedle (circles, upper curve) and a 30G needle (squares, lower curve) into $3 \%$ agarose gel. Both force curves are monotonically increasing, showing that only agarose gel is not sufficient to mimic skin

To study the three-dimensional (3D) insertion process into the gel, we performed two sets of experiments. In the first set, we obtained data from the plane of insertion $(x y)$, and in the second set we obtained out-of-plane data ( $y z$ ). In the first set of experiments, a microneedle was inserted into the gel to three fixed displacements, namely, $500 \mu \mathrm{m}$, $1000 \mu \mathrm{m}$ and $4 \mathrm{~mm}$. After insertion and retraction, all with the same needle, the insertion holes are imaged using the E-SEM. Figure 1(e) shows the results. Note that the letters in the image indicate the orientation of the needle during the insertion, see Figure 1(g) for nomenclature. The needle pushes the gel to the upper right corner in the insertion holes owing to the angle of the bevel plane. The figure shows that with increasing displacement the gel is pushed further away. For the $4000 \mu \mathrm{m}$ displacement, the occurrence of a mode-I crack (opening) is indicated by white arrows (Misra, 2010).

In the second set of experiments, the needle was inserted into the gel and remained inside the gel until a 3D image had been made using confocal microscopy. Figure 1(f) shows such a 3D image of a microneedle inside the agarose gel. The microneedle has been made transparent to visualize the cracks in the gel. The black arrow indicates a mode-II crack (rupture). The letter B indicates the needle orientation.

Figure 5 shows typical force curves of a microneedle and a 30G needle being inserted into 3\% agarose gel. The curves do not exhibit distinct peaks; the force increases monotonically. These curves illustrate that gel only is not sufficient to mimic skin, because previously reported insertion force curves of hypodermic needles into human skin show a distinct maximum in the force when the needle penetrates the tougher, outer skin layers (Shergold, 2005). Therefore, we added a layer of polyurethane foil to mimic the toughness of the skin better.

\subsection{Double-Layer System-Agarose Gel with Polyurethane Foil}

We performed insertion-force experiments on the double-layer skin simulant consisting of 3\% agarose gel and a layer of $80-\mu \mathrm{m}$-thick polyurethane foil on top of the gel. The results for the microneedle and the $30 \mathrm{G}$ needle are plotted in Figures 6 and 7, respectively. The speeds are $50 \mu \mathrm{m} \mathrm{s}^{-1}, 100 \mu \mathrm{m} \mathrm{s}^{-1}, 250 \mu \mathrm{m} \mathrm{s}^{-1}$ and $500 \mu \mathrm{m} \mathrm{s}^{-1}$ for the microneedles and $100 \mu \mathrm{m} \mathrm{s}^{-1}, 250 \mu \mathrm{m} \mathrm{s}^{-1}$, and $500 \mu \mathrm{m} \mathrm{s}^{-1}$ for the $30 \mathrm{G}$ needles. 


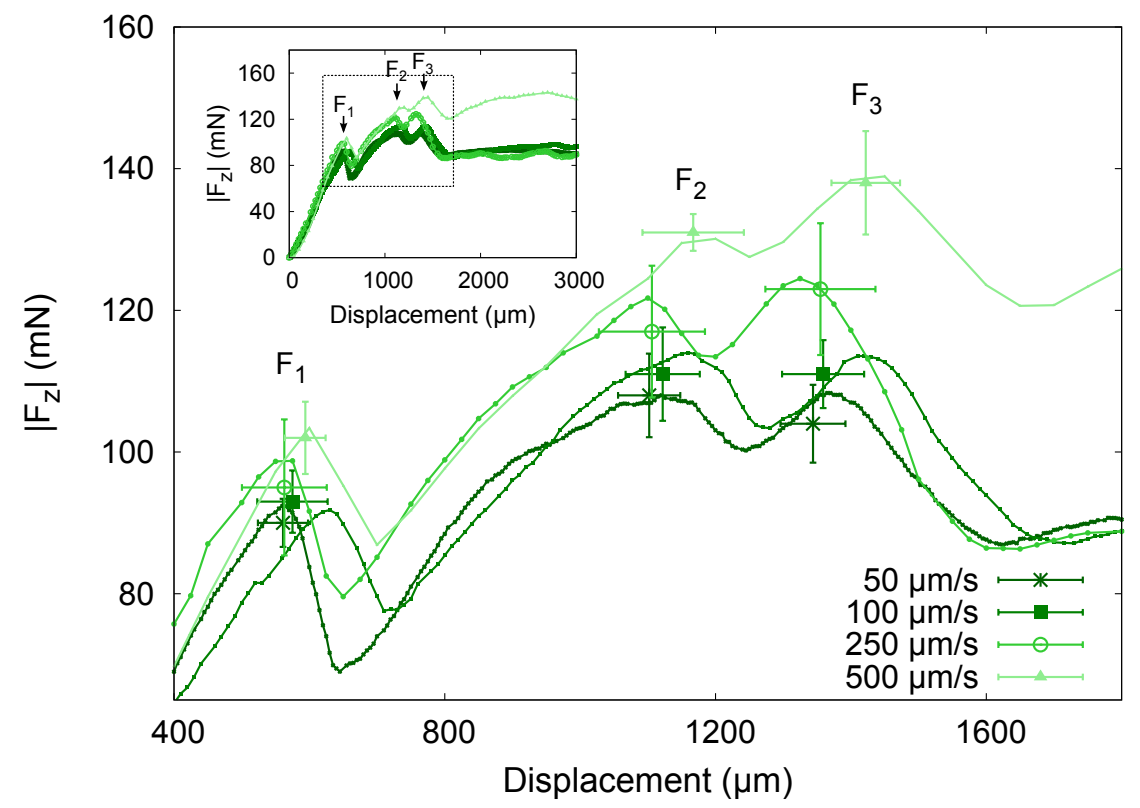

Figure 6. Insertion force-displacement curves of microneedles in 3\% agarose gel with polyurethane foil at different speeds. Each curve represents a typical single experiment at one particular speed; the data points are the mean values of the $F_{1}, F_{2}$ and $F_{3}$ peaks for nine insertion curves including error bars showing the standard deviations. The different speeds are $50 \mu \mathrm{m} \mathrm{s}^{-1}$ (blue), $100 \mu \mathrm{m} \mathrm{s}^{-1}$ (green), $250 \mu \mathrm{m} \mathrm{s}^{-1}$ (red) and $500 \mu \mathrm{m} \mathrm{s}^{-1}$ (brown). The inset shows the same curves for the full displacement used during the experiments

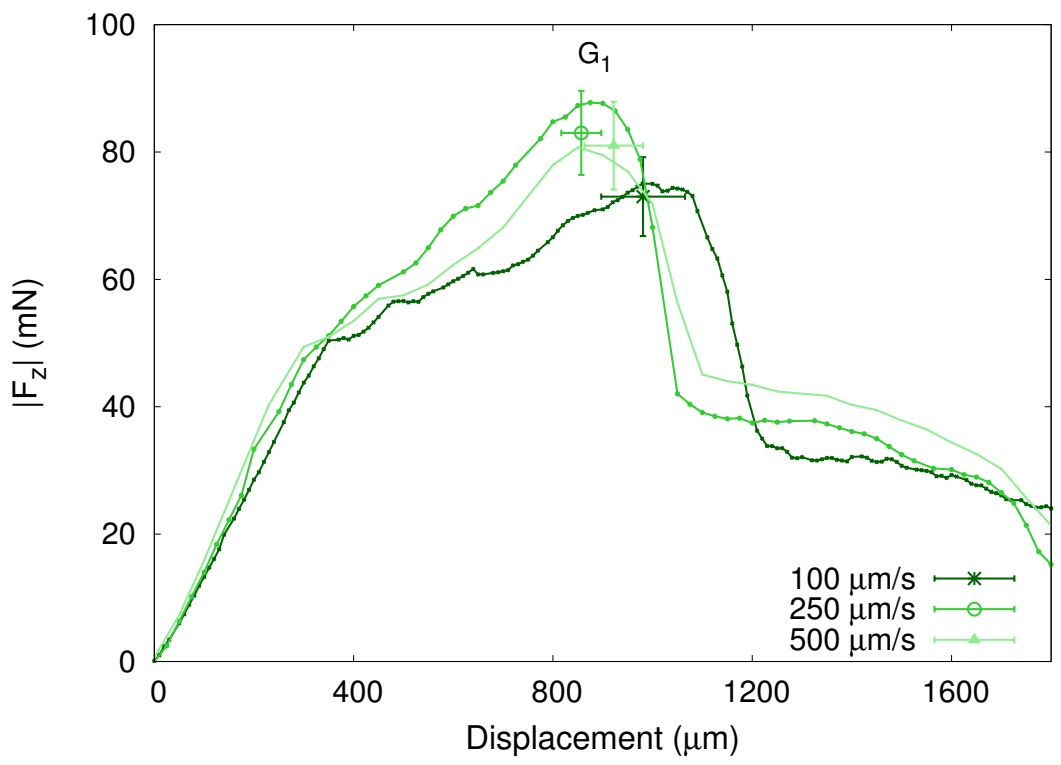

Figure 7. Insertion force-displacement curves of 30G needles in $3 \%$ agarose gel with polyurethane foil at different speeds. Each curve represents a typical single experiment at one particular speed; the data point marked $G_{1}$ is the mean value of the peak for nine insertion curves including error bars showing the standard deviations.

The different speeds are $100 \mu \mathrm{m} \mathrm{s}^{-1}, 250 \mu \mathrm{m} \mathrm{s}^{-1}$ and $500 \mu \mathrm{m} \mathrm{s}^{-1}$ 


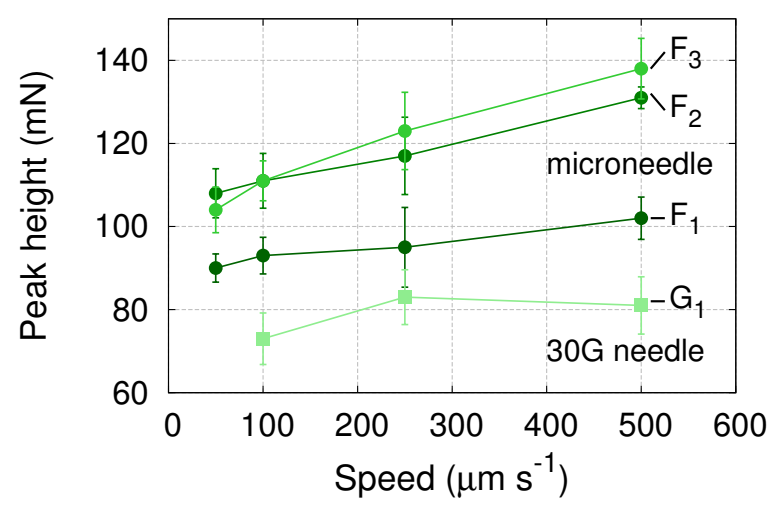

Figure 8. Comparison of the height of the force peaks for the $30 \mathrm{G}$ needles (squares) and microneedles (circles) at various insertion speeds. The error bars show standard deviation. Data reorganized from Figures 6 and 7

The plots show one typical insertion force curve out of a total of nine curves. In all force curves of the microneedles three maxima (force peaks) are identified and termed $F_{1}, F_{2}$ and $F_{3}$ peak. The peaks are indicated in the graphs. There is a gradual increase of the insertion forces with increasing speed. The trend is visualized in figure by plotting the height of the force peaks during insertion for both microneedles and $30 \mathrm{G}$ needles at the various speeds. $F_{1}, F_{2}$ and $F_{3}$ increase with increasing speed. The increase of the $F_{3}$ peak is higher than the increase of the $F_{1}$ peak. At higher speeds the skin simulant has less time to recover from the elastic deformation, which could lead to this additional increase in the peak force. The microneedles have a higher maximum force, on average 1.5 times that of the $30 \mathrm{G}$ needles. The cross-sectional area of the microneedles is 1.7 times that of the $30 \mathrm{G}$ needle. Also, the forces at larger displacement values $(>2 \mathrm{~mm})$ are substantially higher for the microneedles than for the $30 \mathrm{G}$ needles. We attribute this difference to a coating that reduces friction on the $30 \mathrm{G}$ needles, whereas the microneedles are not coated. The difference in the shape of the shaft, i.e., circular or rectangular, can also lead to different insertion forces at larger insertion depth, because of the potential lower friction for rounded shafts. The overall trend with increasing speed is that a higher force is needed to insert the needles. For the range of speeds that we used, this relation is in agreement with (Okamura, 2004).

All 30G-needle insertion-force curves feature a distinct peak, and the points marked by $G_{1}$ in Figure 7 represent the mean value of the peak force. The general trend of the curves is in agreement with previous reports (Mayer, 2009; Shergold, 2005), showing that the skin simulant behaves as expected. When the maximum force is reached at $G_{1}$, an opening is created, propagating a crack into the tissue (Mahvash, 2010). At approximately 2 mm needle displacement, the full length of the bevel (approximately $1.5 \mathrm{~mm}$ long) penetrated the back side of the foil. The force curve flattens out, and is then governed by the force needed to propagate the needle into the gel and to overcome friction forces.

\subsection{Double-Layer System-Insertion-Force Analysis}

The low values for the standard deviation in Figures 6 and 7 indicate that the results are consistent and that the insertion force can be used for systematic analysis of a series of experiments that relate the geometry of the microneedle to the force curves. Before every insertion experiment, the needle was translated until it was in contact with the foil. From this point, the needle was displaced by a fixed amount into the double-layer skin simulant. Next, the needle was retracted. The series was continued by increasing the displacement in steps of $200 \mu \mathrm{m}$. The experiments were performed for both the microneedle and the $30 \mathrm{G}$ needle. The two force curves with the largest insertion depth are shown in figures and, the other force curves are not shown. At these insertion depths, all characteristic peaks appeared in the force curve. Both series were obtained with the same needle and skin simulant to maintain consistency. The insertion speed is $100 \mu \mathrm{ms}^{-1}$.

After the insertion experiments, the foil was carefully removed from the agarose gel and placed in the SEM. Then every insertion hole was imaged from the front and back side of the foil, see Figures 10 and 12 . The front side of the foil is defined as the side where the needles first enter the foil.

Analysis of the SEM images allows us to identify the different phases of insertion of the needles into the skin simulant. The phases are indicated in figures and and the gray circles on the $x$-axis correspond to the displacements 
of all experiments.

In the case of the microneedle, starting from zero displacement, the needle initially deforms the foil. We note that both elastic and plastic deformation occured, as shown by the SEM image at $400 \mu \mathrm{m}$ and as observed visually during the experiment. The SEM image taken at $400 \mu \mathrm{m}$ of the back side of the foil reveals that the needle tip has already punctured the foil. This means that the $F_{1}$ peak is not caused by the tip $\left(Z_{0}\right)$ piercing the foil. Moreover, there are no traces of foil piercing by the tip in the force curve. The $F_{1}$ peak is reached at about $500 \mu \mathrm{m}$ displacement. The SEM image of the front side of the foil at $600 \mu \mathrm{m}$ insertion shows that the horizontal cut is complete. Even though the needle is inserted further in subsequent experiments, the width of the cut does not increase further.

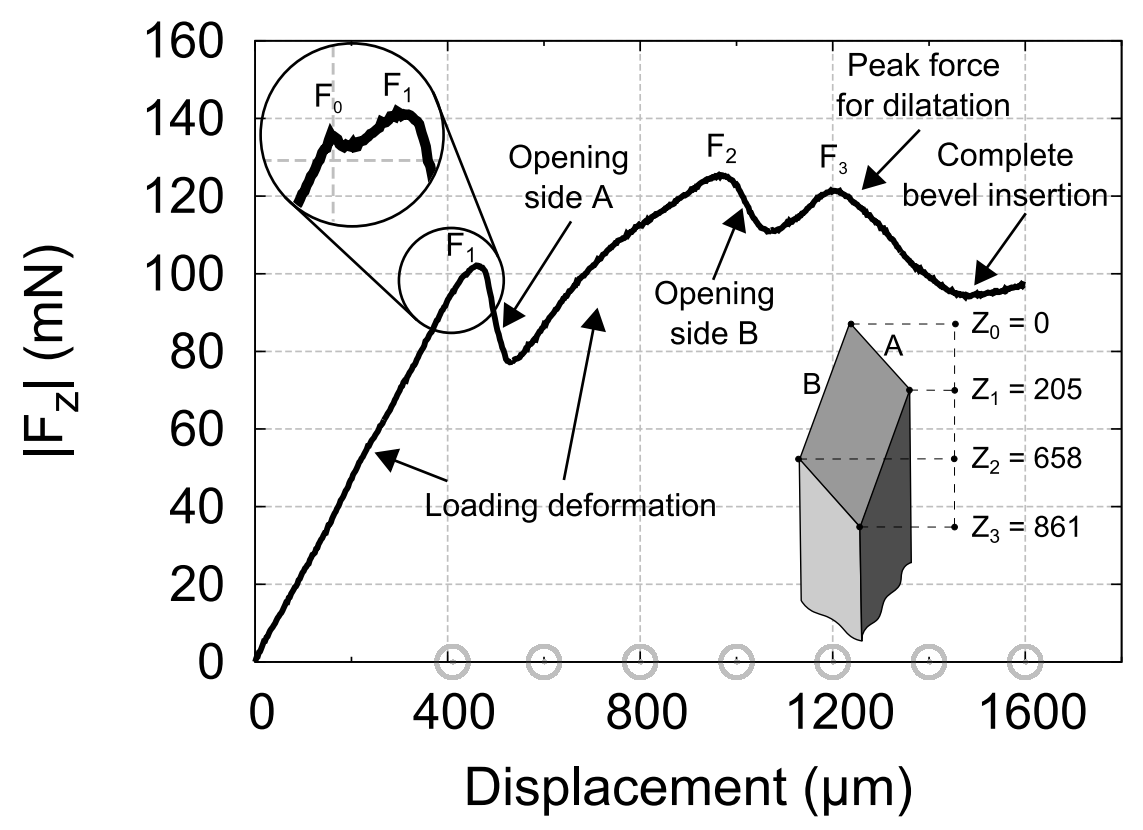

Figure 9. Force-displacement curve of a microneedle-insertion experiment. The force characteristics are indicated in the graph. The encircled inset shows that an additional small peak appears if a blunt needle tip is used. The gray circles on the $x$-axis indicate the amount of displacement of the other insertion experiments in the series
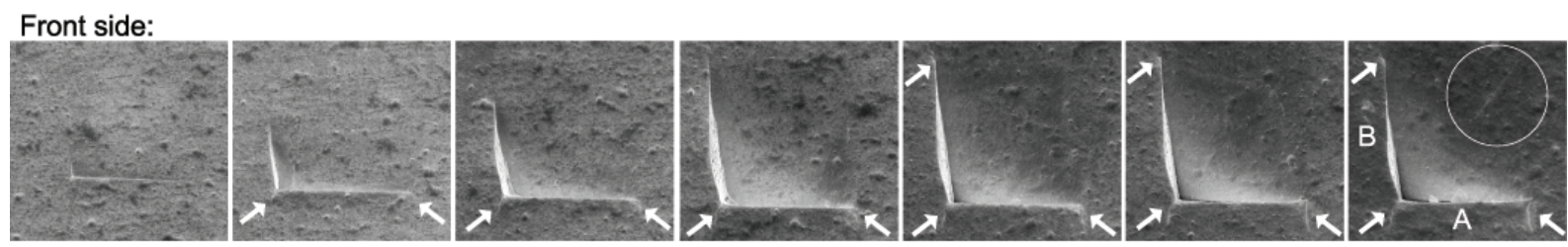

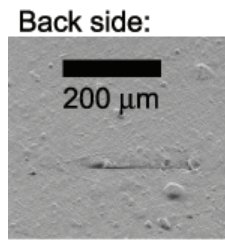

$400 \mu \mathrm{m}$

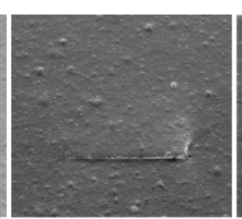

$600 \mu \mathrm{m}$

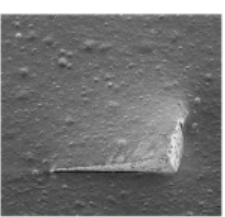

$800 \mu \mathrm{m}$

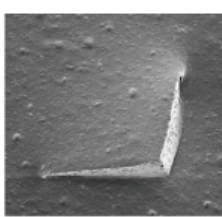

$1000 \mu \mathrm{m}$

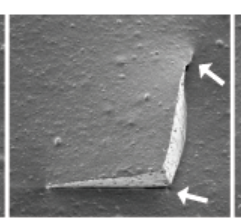

$1200 \mu \mathrm{m}$

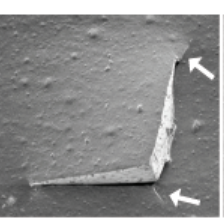

$1400 \mu \mathrm{m}$

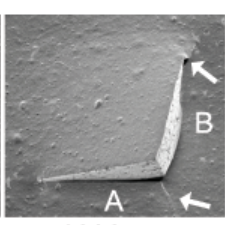

$1600 \mu \mathrm{m}$

Figure 10. Series of SEM images of insertion holes created by a microneedle in polyurethane foil at identical scale. The top row shows the front side of the foil with the viewing direction equal to the surface normal. The bottom row shows the back side of the foil under an oblique view of $20^{\circ}$ with respect to the surface normal. The distances under each pair of images correspond to the displacement of the needle into the skin simulant. A mode-I crack (opening) is indicated by a white arrow 


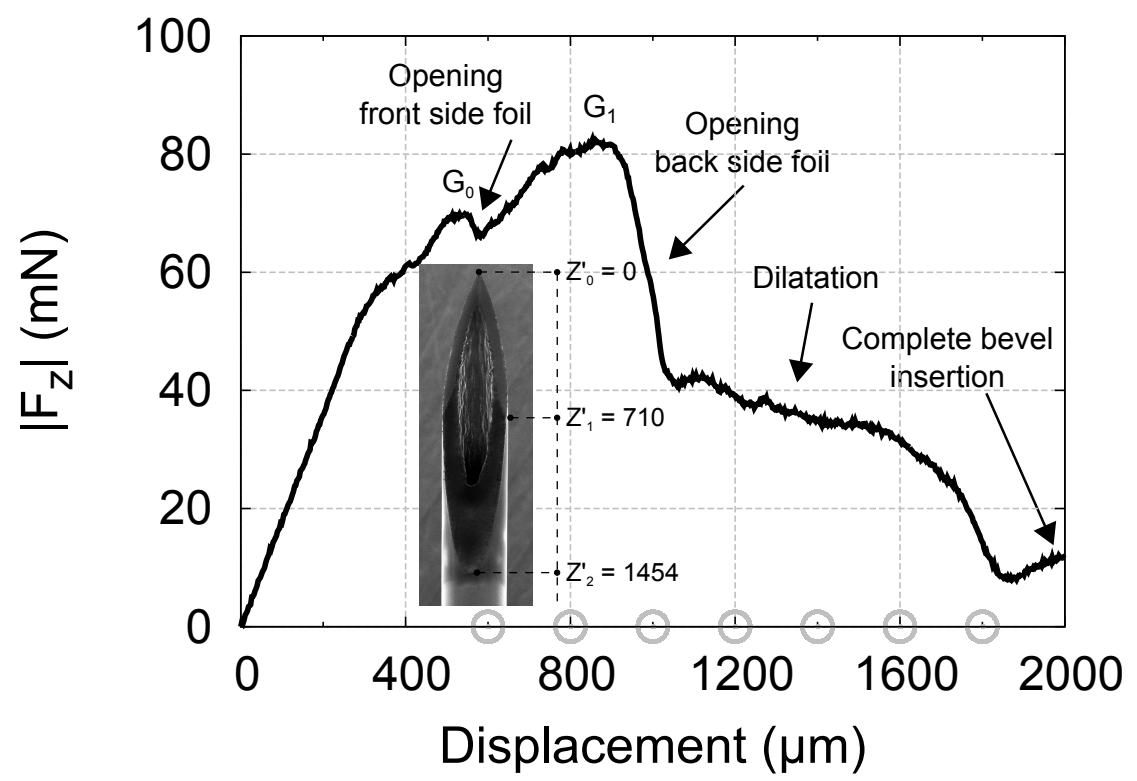

Figure 11. Force-displacement curve of a $30 \mathrm{G}$ needle-insertion experiment. The force characteristics are indicated in the graph. The inset shows a SEM image of tip of the $30 \mathrm{G}$ needle. The gray circles on the $x$-axis indicate the amount of displacement of the other insertion experiments in the series

Front side:

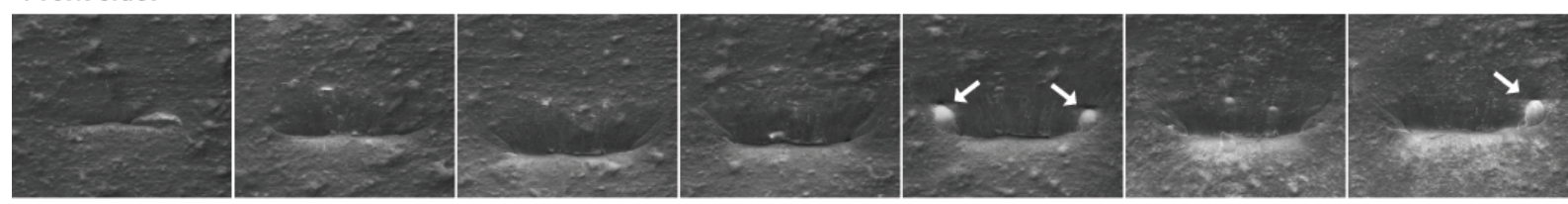

Back side:

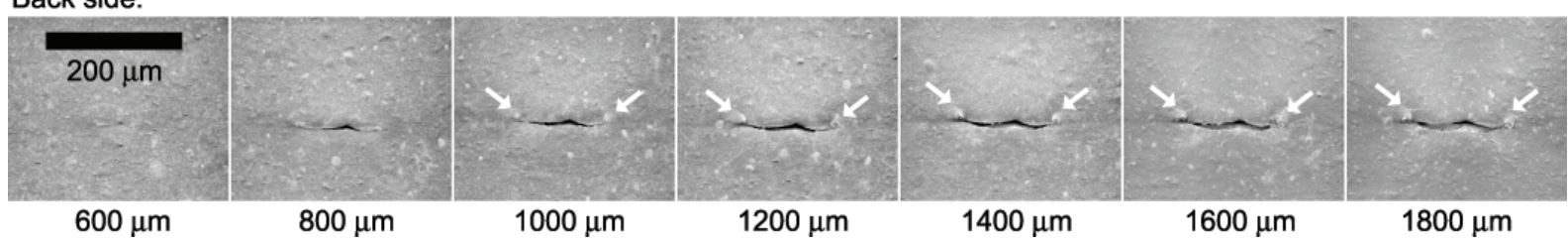

Figure 12. Series of SEM images of insertion holes created by a $30 \mathrm{G}$ needle in polyurethane foil at identical scale. The top row shows the front side of the foil. The bottom row shows the back side of the foil. All viewing directions are equal to the surface normal. The distances under each pair of images correspond to the displacement of the needle into the skin simulant. A mode-I crack (opening) is indicated by a white arrow

From this observation we conclude that the $F_{1}$ peak is caused by the completion of the horizontal cut, created by side $A$ of the needle $\left(Z_{0}-Z_{1}\right.$, refer to the inset in Figure 9$)$. The decrease in the insertion force curve is caused by a mode-I crack (opening) inside the foil, which, in turn, is triggered by the increase of strain when corner $Z_{1}$ of the needle is inserted into the foil. Note that the openings (cracks) at the corners are visible in a number of the SEM images, on both the front and back side of the foil.

An additional experiment using a blunt needle (tip diameter of approximately $15 \mu \mathrm{m}$ ) supports this conclusion. In figure, an inset shows the corresponding portion of the force curve around the $F_{1}$ peak. Before the $F_{1}$ peak there is an $F_{0}$ peak visible, which indicates that the blunt needle tip $\left(Z_{0}\right)$ punctures the foil. These data show that a blunt needle can be distinguished from a sharp needle.

After the opening of the foil caused by side A of the needle, the force increases again while the needle cuts with side $\mathrm{B}\left(Z_{0}-Z_{2}\right)$. Also, the skin simulant is elastically deformed by the increase in force. Around $1000 \mu \mathrm{m}$ needle displacement, the $F_{2}$ peak appears, corresponding to the complete insertion of side B. Again, a rupturing of the foil takes place, best visible in the SEM images of the back side of the foil at 1200, 1400 and $1600 \mu \mathrm{m}$ (upper right corners). 
Remarkably, the cutting phases have stopped at this point. The rest of the needle is inserted by dilatation of the insertion hole. The free-hanging part of the foil is pushed into the gel, evidenced by a scratch mark on the front side of the foil caused by corner $Z_{3}$. The mark runs from the upper right corner towards the center of the SEM image and is indicated by a white circle in the $1600-\mu \mathrm{m}$-image (Figure 10). The $F_{3}$ peak is the maximum force needed to dilate the insertion hole.

The $30 \mathrm{G}$ needle shows two peaks in the force curve, termed $G_{0}$ peak and $G_{1}$ peak, see figure. The $G_{0}$ peak is reached at approximately $550 \mu \mathrm{m}$ displacement. This displacement is approximated by the $600 \mu \mathrm{m}$ SEM images (in Figure 12). The SEM image of the front side of the foil shows that the maximum width of the cut is reached. The $G_{1}$ peak is reached at approximately $950 \mu \mathrm{m}$ displacement. In the SEM images of the back side of the foil, it can be seen that at $1000 \mu \mathrm{m}$ displacement the width of the cut is maximum. Also, a mode-I crack (opening) is visible at both sides of the cut, indicated by a white arrow in Figure 12. From these observations, we conclude that the large drop in force is caused by an opening of the foil. We hypothesize that the $G_{1}$ peak occurs when point $Z_{1}^{\prime}$ of the needle (the transition point between the two different bevel angles of the $30 \mathrm{G}$ needle) pierces the foil.

When comparing the insertion holes made by the microneedle in Figure 10 and those made by the $30 \mathrm{G}$ needle in Figure 12 it is noticed that the $30 \mathrm{G}$ needle only makes one horizontal cut, and the microneedle makes two cuts before the remainder of the needle is inserted by dilation and additional crack formation. The insertion holes of the $30 \mathrm{G}$ close up after removal of the needle, whereas the insertion holes of the microneedle leave a part of the foil partially hanging free. The cutting of the microneedle exhibits well-defined lines, indicating that the sides of the microneedle are sharper than the sides of the $30 \mathrm{G}$ needle. Also, the force peaks $\left(F_{1}\right.$ through $\left.F_{3}\right)$ in the insertion curve of the microneedle can be directly related to the sharp and well-defined corners $\left(Z_{1}\right.$ through $Z_{3}$ ), whereas the $30 \mathrm{G}$ needle geometry is rounded and has a more gradual change in shape when inserting from tip to shaft.

This analysis clearly illustrates that characterization methods in combination with a skin simulant allow a detailed study of the insertion holes and that the force curve can be related to the geometry of the microneedle. Also, Figures 10 and 12 show that the results are consistent within each series owing to the repeatability of the experiment. This repeatability makes reliable characterization of (micro)needles possible, e.g., a blunt tip will be recognized in the force curve.

\section{Conclusions}

Methods to characterize the interaction between microneedles and soft tissue have been introduced. A skin simulant consisting of 3\% agarose gel and a polyurethane foil has been used to perform a detailed study of the insertion characteristics of microneedles and hypodermic needles. Insertion-force recordings have been combined with imaging techniques, such as confocal microscopy with epifluorescence and electron microscopy, to study the interaction of needles with the skin simulant. The images allow the interpretation of the insertion-force curves and make it possible to relate the curve to the needle geometry. The characterization methods demonstrated in combination with the skin simulant allow the characterization of microneedles and enable comparisons with other needle designs, such as the hypodermic 30G needle also used in this study. Mode-I (opening) and mode-II (rupture) cracks can be identified in the images of the gel and foil using the microscopy techniques mentioned. The force peaks in the insertion curves can be related to specific features of the needle geometry. In the case of the microneedle three peaks have been distinguished in the force curve that directly relate to three sharp corners of the microneedle.

The maximum insertion force of the microneedles increases with insertion speed, to a limited extent of $14 \%$ in a speed range of $50-500 \mu \mathrm{ms}^{-1}$. For the $30 \mathrm{G}$ needles, a $12 \%$ increase in maximum insertion force is measured in a speed range of $100-500 \mu \mathrm{ms}^{-1}$. This study will help optimize microneedle design and developing microneedletissue interaction models.

\section{Acknowledgments}

The authors gratefully acknowledge several colleagues from U-Needle B.V. and from the University of Twente for their contribution to this work. In particular we thank A. Jahya and H. van Wolferen for help with imaging, A. Been for constructing the insertion setup, R. Visser for help with the stress-strain measurements, M. van Barneveld for useful discussion, and M. van de Vorle for needle fabrication. This study is supported by the Dutch Ministry of Economic Affairs, region Overijssel, region Gelderland, under the programme Nano4Vitaliy.

\section{References}

Campbell, P. K., Jones, K. E., Huber, R. J., Horch, K. W., \& Normann, R. A. (1991). A silicon-based, threedimensional neural interface: Manufacturing processes for an intracortical electrode array. IEEE Trans. Biomed. 
Eng., 38(8), 758-768. http://dx.doi.org/10.1109/10.83588

Davis, S. P., Landis, B. J., Adams, Z. H., Allen, M. G., \& Prausnitz, M. R. (2004). Insertion of microneedles into skin: Measurement and prediction of insertion force and needle fracture force. J. Biomech., 37(8), 1155-1163. http://dx.doi.org/10.1016/j.jbiomech.2003.12.010

Diridollou, S., Patat, F., Gens, F., Vaillant, L., Black, D., Lagarde, J. M., \& Berson, M. (2000). In vivo model of the mechanical properties of the human skin under suction. Skin Res. Technol., 6(4), 214-221. http://dx.doi.org/10.1034/j.1600- 0846.2000.006004214.x

Donnelly, R. F., Raj Singh, T. R., \& Woolfson, A. D. (2010). Microneedle-based drug delivery systems: Microfabrication, drug delivery, and safety. Drug Deliv., 17(4), 187-207. http://dx.doi.org/10.3109/10717541003667798

Doraiswamy, A., Ovsianikov, A., Gittard, S. D., Monteiro-Riviere, N. A., Crombez, R., Montalvo, E., \& Narayan, R. J. (2010). Fabrication of microneedles using two photon polymerization for transdermal delivery of nanomaterials. J. Nanosci. Nanotechnol., 10(10), 6305-6312. http://dx.doi.org/10.1166/jnn.2010.2636

Egekvist, H., Bjerring, P., \& Arendt-Nielsen, L. (1999). Pain and mechanical injury of human skin following needle insertions. Eur. J. Pain, 3(1), 41-49. http://dx.doi.org/10.1016/S1090-3801(99)90187-8

Gill, H. S., Denson, D. D., Burris, B. A., \& Prausnitz, M. R. (2008). Effect of microneedle design on pain in human volunteers. Clin. J. Pain, 24(7), 585-594. http://dx.doi.org/10.1097/AJP.0b013e31816778f9

Gill, H. S., \& Prausnitz, M. R. (2007). Coated microneedles for transdermal delivery. J. Control. Release, 117(2), 227-237. http://dx.doi.org/10.1016/j.jconrel.2006.10.017

Henry, S., McAllister, D. V., Allen, M. G., \& Prausnitz, M. R. (1998). Microfabricated microneedles: A novel approach to transdermal drug delivery. J. Pharm. Sci., 87(8), 922-925. http://dx.doi.org/10.1021/js980042+

Hilt, J. Z., \& Peppas, N. A. (2005). Microfabricated drug delivery devices. Int. J. Pharm., 306(1-2), 15-23. http://dx.doi.org/10.1016/j.ijpharm.2005.09.022

Jiang, J., Gill, H. S., Ghate, D., McCarey, B. E., Patel, S. R., Edelhauser, H. F., \& Prausnitz, M. R. (2007). Coated microneedles for drug delivery to the eye. Invest. Ophthalmol. Vis. Sci., 48(9), 4038-4043. http://dx.doi.org/10.1167/iovs.07- 0066

Kim, S., Shetty, S., Price, D., \& Bhansali, S. (2006). Skin penetration of silicon dioxide microneedle arrays. In Conf. Proc. IEEE Eng. Med. Biol. Soc.(pp. 4088-4091). New York, NY. http://dx.doi.org/10.1109/IEMBS.2006.260142

Lee, J. W., Park, J.-H., \& Prausnitz, M. R. (2008). Dissolving microneedles for transdermal drug delivery. Biomaterials, 29(13), 2113-2124. http://dx.doi.org/10.1016/j.biomaterials.2007.12.048

Mahvash, M., \& Dupont, P. E. (2010). Mechanics of dynamic needle insertion into a biological material. IEEE Trans. Biomed. Eng., 57(4), 934-943. http://dx.doi.org/10.1109/TBME.2009.2036856

Mayer, G., Knappertz, V., \& Kinast, P. (2009). Needles: a comparison study. Med. Device Technol., 20(7), 32-34.

McAllister, D. V., Wang, P. M., Davis, S. P., Park, J.-H., Canatella, P. J., Allen, M. G., \& Prausnitz, M. R. (2003). Microfabricated needles for transdermal delivery of macromolecules and nanoparticles: Fabrication methods and transport studies. Proc. Natl. Acad. Sci. U. S. A., 100(SUPPL. 2), 13755-13760. http://dx.doi.org/10.1073/pnas.2331316100

Misra, S., Reed, K. B., Schafer, B. W., Ramesh, K. T., \& Okamura, A. M. (2010). Mechanics of flexible needles robotically steered through soft tissue. Int. J. Rob. Res., 29(13), 1640-1660. http://dx.doi.org/10.1177/0278364910369714

Okamura, A. M., Simone, C., \& OLeary, M. D. (2004). Force modeling for needle insertion into soft tissue. IEEE Trans. Biomed. Eng., 51(10), 1707-1716. http://dx.doi.org/10.1109/TBME.2004.831542

Park, J.-H., Allen, M. G., \& Prausnitz, M. R. (2005). Biodegradable polymer microneedles: Fabrication, mechanics and transdermal drug delivery. J. Control. Release, 104(1), 51-66. http://dx.doi.org/10.1016/j.jconrel.2005.02.002

Park, J.-H., Allen, M. G., \& Prausnitz, M. R. (2006). Polymer microneedles for controlled-release drug delivery. Pharm. Res., 23(5), 1008-1019. http://dx.doi.org/10.1007/s11095-006-0028-9 
Roxhed, N., Gasser, T. C., Griss, P., Holzapfel, G. A., \& Stemme, G. (2007). Penetration-enhanced ultrasharp microneedles and prediction on skin interaction for efficient transdermal drug delivery. J. Microelectromech. Syst., 16(6), 1429-1440. http://dx.doi.org/10.1109/JMEMS.2007.907461

Shergold, O. A., \& Fleck, N. A. (2005). Experimental investigation into the deep penetration of soft solids by sharp and blunt punches, with application to the piercing of skin. J. Biomech. Eng., 127(5), 838-848. http://dx.doi.org/10.1115/1.1992528

Stoeber, B., \& Liepmann, D. (2005). Arrays of hollow out-of-plane microneedles for drug delivery. J. Microelectromech. Syst., 14(3), 472-479. http://dx.doi.org/10.1109/JMEMS.2005.844843

Wang, P. M., Cornwell, M., Hill, J., \& Prausnitz, M. R. (2006). Precise microinjection into skin using hollow microneedles. J. Invest. Dermatol., 126(5), 1080-1087. http://dx.doi.org/10.1038/sj.jid.5700150

Wang, P. M., Cornwell, M., \& Prausnitz, M. R. (2005). Minimally invasive extraction of dermal interstitial fluid for glucose monitoring using microneedles. Diabetes Technol. Ther., 7(1), 131-141. http://dx.doi.org/10.1089/dia.2005.7.131

Zhang, P., Dalton, C., \& Jullien, G. A. (2009). Design and fabrication of MEMS-based microneedle arrays for medical applications. Microsyst. Technol., 15(7), 1073-1082. http://dx.doi.org/10.1007/s00542-009-0883-5

\section{Copyrights}

Copyright for this article is retained by the author(s), with first publication rights granted to the journal. This is an open-access article distributed under the terms and conditions of the Creative Commons Attribution license (http://creativecommons.org/licenses/by/3.0/). 